\title{
Quasi-classical trajectory study of the adiabatic reactions occurring on the two lowest-lying electronic states of the $\mathrm{LiH}_{2}{ }^{+}$system
}

\author{
Ilaria Pino, ${ }^{* a}$ Rocco Martinazzo ${ }^{b}$ and Gian Franco Tantardini ${ }^{b c}$ \\ Received 4th April 2008, Accepted 10th June 2008 \\ First published as an Advance Article on the web 23rd July 2008 \\ DOI: $10.1039 / \mathbf{b 8 0 5 7 5 0 h}$
}

Quasi-classical trajectory calculations have been performed on the adiabatically allowed reactions taking place on the two lowest-lying electronic states of the $\mathrm{LiH}_{2}{ }^{+}$system, using the ab initio potential energy surfaces of Martinazzo et al. (J. Chem. Phys., 2003, 119, 11 241). These reactions comprise: (i) the exoergic $\mathrm{H}_{2}$ and $\mathrm{H}_{2}{ }^{+}$formation occurring through $\mathrm{LiH}^{+}+\mathrm{H}$ and $\mathrm{LiH}+\mathrm{H}^{+}$ collisions in the ground and in the first electronically excited state, respectively; (ii) the endoergic (ground state) $\mathrm{LiH}^{+}$dissociation induced by collisions with $\mathrm{H}$ atoms; and (iii) the endoergic (excited state) $\mathrm{Li}+\mathrm{H}_{2}{ }^{+} \rightarrow \mathrm{LiH}+\mathrm{H}^{+}$reaction. The topic is of relevance for a better understanding of the lithium chemistry in the early universe. Thermal rate constants for the above reactions have been computed in the temperature range 10-5000 K and found in reasonably good agreement with estimates based on the capture model.

\section{Introduction}

Much effort has been spent in the recent years to understand how the first molecules formed in the early universe and how their abundance evolved during the so-called post-recombination era. The aim is to explain the mechanism of the density fluctuations that led to the first macroscopic objects formation. ${ }^{1-6}$ According to the standard Big Bang nucleosynthesis model, the early universe was chemically very simple and only electrons, low energy photons and the lightest nuclei H, D, He and Li took part in a relatively simple chemical reaction network in an entirely gaseous phase. ${ }^{7-9}$ Despite the low lithium cosmic abundance, lithium chemistry in the early universe ${ }^{9,10}$ has recently received some attention since it has been recognized that important contributions to the cosmic background radiation spectrum could have arisen from the high dipole moment carriers such as $\mathrm{LiH}$, if their abundance had been enough. ${ }^{11} \mathrm{LiH}$ is known to be formed through radiative association and depleted in collisions with the abundant hydrogen atoms to form $\mathrm{H}_{2}$. However, its abundance is still uncertain because of the poor information about the processes it is involved in. ${ }^{9,10}$. One complicating factor is the possible importance of the ionic chemistry. Indeed, because of the low ionization potential of Li-bearing species, the abundance of $\mathrm{LiH}^{+}$can overcome that of its neutral counterpart. ${ }^{9}$ In this "lithium chemistry" 9,10 a number of processes either lack information about the values of the corresponding rate constants or have only estimated values for them. They are listed in Table 1 for completeness. The computation and analytic fitting of accurate adiabatic potential energy surfaces (PESs) of the two lowest-lying singlet electronic states of

a Dipartimento di Chimica, Università "Federico II", Complesso Monte S. Angelo, via Cintia, 80126 Napoli, Italy.

E-mail: ilaria.pino@unina.it

${ }^{b}$ Dipartimento di Chimica Fisica ed Elettrochimica, Università degli

Studi di Milano, via Golgi 19, 20133 Milano, Italy

${ }^{c}$ CNR Istituto di Scienze e Tecnologie Molecolari, via Golgi 19,

20133 Milano, Italy
$\mathrm{LiH}_{2}{ }^{+12-15}$ have recently shed a new light on reactions (4-11) of Table 1: the lack of conical intersections between the ground and the first excited states of $\mathrm{LiH}_{2}{ }^{+}$makes the charge exchange reactions (4-7) forbidden, ${ }^{16}$ whereas reactions 4 and 7 are generally taken into account in evolutionary models. A conical intersection due to charge exchange between the two hydrogen atoms does occur in this system but it is between the first and the second excited states with an energy too high to be of any interest. ${ }^{15}$ In this work we use the three-dimensional potential energy surfaces computed and fitted by Martinazzo et al. ${ }^{15}$ on the $\mathrm{LiH}_{2}{ }^{+}$system in conjunction with quasi-classical trajectory (QCT) methods to compute the rate constants for reactions $(8-11)$ of Table 1 . In particular, the weakly endoergic reactions (10-11) have never been taken into account in chemical-physics models aimed to compute $\mathrm{LiH} / \mathrm{LiH}^{+}$abundances ${ }^{10}$ in spite of their possible relevance due to long-range interactions between the reactants. A scheme of the energetics of the reactions relevant to this work is shown in Fig. 1, based on the data of ref. 15. The ground-state $\mathrm{LiH}^{+}+\mathrm{H} \rightarrow \mathrm{Li}^{+}+\mathrm{H}_{2}$ reaction is a barrierless, strongly exoergic process. This is due to the low binding energy

Table 1 Some ionic reactions in the lithium chemistry. Estimates by Stancil et al. ${ }^{42}$

\begin{tabular}{lll}
\hline No. & Reaction & $k / \mathrm{cm}^{3} \mathrm{~s}^{-1}$ \\
\hline$(1)$ & $\mathrm{LiH}^{+}+\mathrm{e} \rightarrow \mathrm{Li}+\mathrm{H}$ & $3.8 \times 10^{-7} \mathrm{~T}^{-0.47}$ \\
$(2)$ & $\mathrm{Li}+\mathrm{H}^{-} \rightarrow \mathrm{LiH}+\mathrm{e}$ & $4.0 \times 10^{-10}$ \\
$(3)$ & $\mathrm{Li}^{-}+\mathrm{H} \rightarrow \mathrm{LiH}+\mathrm{e}$ & $4.0 \times 10^{-10}$ \\
$(4)$ & $\mathrm{LiH}^{+}+\mathrm{H} \rightarrow \mathrm{LiH}+\mathrm{H}^{+}$ & $1.0 \times 10^{-11} \exp \left(-\frac{67900}{T_{g}}\right)$ \\
$(5)$ & $\mathrm{LiH}+\mathrm{H}^{+} \rightarrow \mathrm{LiH}^{+}+\mathrm{H}$ & - \\
$(6)$ & $\mathrm{LiH}+\mathrm{H}^{+} \rightarrow \mathrm{Li}^{+}+\mathrm{H}_{2}$ & - \\
$(7)$ & $\mathrm{LiH}^{+}+\mathrm{H} \rightarrow \mathrm{Li}^{+} \mathrm{H}_{2}^{+}$ & $9.0 \times 10^{-10} \exp \left(-\frac{66400}{T_{g}}\right)$ \\
$(8)$ & $\mathrm{LiH}^{+} \mathrm{H}^{+} \rightarrow \mathrm{Li}^{+} \mathrm{H}_{2}^{+}$ & $1.0 \times 10^{-9}$ \\
$(9)$ & $\mathrm{LiH}^{+}+\mathrm{H} \rightarrow \mathrm{Li}^{+}+\mathrm{H}_{2}$ & $3.0 \times 10^{-10}$ \\
$(10)$ & $\mathrm{LiH}^{+}+\mathrm{H} \rightarrow \mathrm{Li}^{+}+\mathrm{H}^{+} \mathrm{H}$ & - \\
$(11)$ & $\mathrm{Li}^{+} \mathrm{H}_{2}^{+} \rightarrow \mathrm{LiH}^{+} \mathrm{H}^{+}$ & -
\end{tabular}




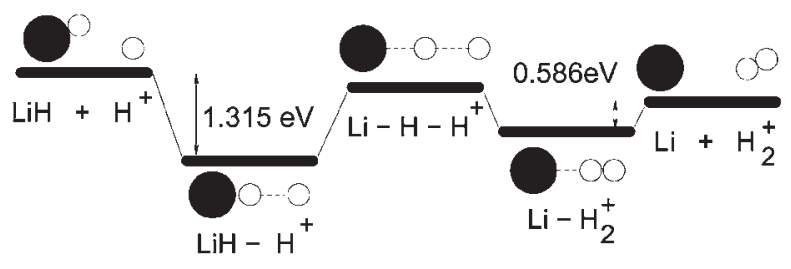

ca. $10 \mathrm{eV}$

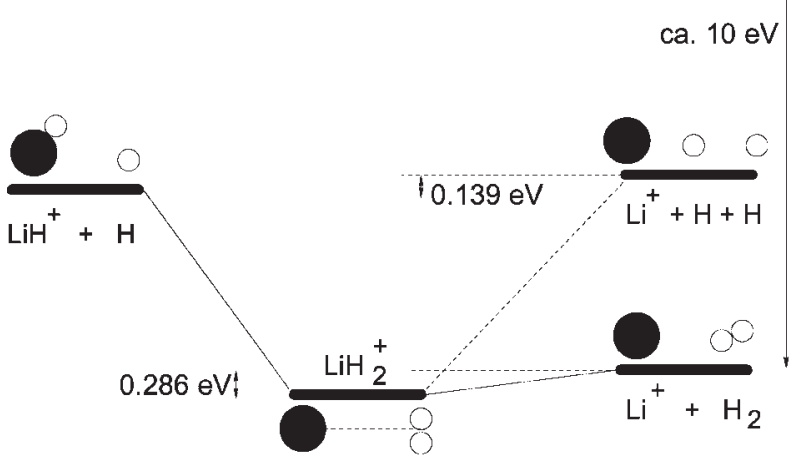

Fig. 1 Energy diagram of the processes considered in this work.

of the $\mathrm{LiH}^{+}$molecule $\left(D_{0}=0.112 \mathrm{eV}\right)$, which makes the threebody break-up channel accessible already at low collision energy. The shallow global minimum corresponds to the weakly bound, $\mathrm{T}$-shaped $\mathrm{Li}^{+} \ldots \mathrm{H}_{2}$ complex. The excited-state potential, on the other hand, shows all the features of a complex system, with two deep wells corresponding to the (linear) electrostatic complexes $\mathrm{LiH} \cdots \mathrm{H}^{+}$and $\mathrm{Li} \cdots \mathrm{H}_{2}{ }^{+}$. Note that the barrier to their interconversion is lower than the highest energy channel $\mathrm{LiH}+\mathrm{H}^{+}$, and therefore the exoergic reaction is barrierless too. The exoergicity of the reactions is $4.365(4.609)$ and $0.217(0.272) \mathrm{eV}$, for the ground and the excited electronic states, respectively, with (without) the zero-point energy corrections. The PESs used in this work are based on more than 11000 ab initio points computed with a multi-reference valence bond approach ${ }^{14,17}$ and corrected with about 600 points calculated with multireference configuration interaction (MRCI) wavefunctions based on complete active space self-consistent-field (CASSCF) reference wavefunctions and a large basis set. ${ }^{15}$ The ground-state potential energy surface has already been employed in a number of studies of the $\mathrm{LiH}_{2}{ }^{+}$ground-state complex, ${ }^{18-20}$ which has recently been subjected to spectroscopic investigations. ${ }^{21,22}$ It was found that the PES is reasonably accurate, despite the fact that its analytic fitting was globally optimized in the configuration space and the fact that the $a b$ initio calculations lacked any correction for the basis set superposition error. More recent theoretical studies, both at the CASSCF-MRCI ${ }^{19}$ and at the full- $\mathrm{CI}^{20}$ level, essentially agree with the results of ref. 15. The excited-state potential has already been used in dynamical calculations of the $\mathrm{LiH}+\mathrm{H}^{+} \rightarrow \mathrm{Li}+\mathrm{H}_{2}{ }^{+}$reactions (direct and reverse $)^{23-25}$ and inelastic scattering. ${ }^{26,27}$ First quantum dynamical studies ${ }^{24}$ surprisingly found that the above exoergic reaction was a dynamically activated process, but recent quantum and quasi-classical trajectory investigations ${ }^{25}$ turned out to be at variance with previous results. The paper is organized as follows. In section 2 we briefly summarize the computational details, while in sections 3 and 4 we discuss the results for the ground and the excited state reactions. In section 5 we summarize and conclude.

\section{Computational details}

We performed quasi-classical trajectory (QCT) calculations $^{28}$ with a three-body adapted version of our parallel gas-surface code TRAJ, ${ }^{29-33}$ see ref. 34 for details. Total energy and angular momentum deviations below $10^{-7}$ atomic units were obtained at a reasonable CPU cost by using a variable step Nordsieck-Gear's predictor-modifier-corrector algorithm. ${ }^{35}$ Trajectory integration was stopped when at least two out of the three molecular separations exceeded $45 \AA$, and further analysis was performed on the closest atomic pair on energetic grounds. In this way, when the break-up channel opens (see section 3) we are able to distinguish diatomic products into stable and quasi-bound molecules trapped by the centrifugal barrier. The initial vibrational phase of the reactant molecule was selected by standard prescriptions, using rovibrational energies calculated via a Colbert-Miller discrete variable representation method. ${ }^{36}$ Cross sections were evaluated on batches of 5000 trajectories for each collision energy and initial rovibrational state, via a Monte Carlo sampling of the impact parameter $b$ within a maximum value $b_{\max }$ estimated from opacity functions calculations at selected collision energies. Opacity functions were obtained by running batches of 500 trajectories for each impact parameter. Thermal rate constants were computed from batches of $10^{4}-10^{5}$ trajectories for each temperature, via Monte Carlo sampling of the initial rovibrational states and collision energies according to the Boltzmann and Maxwell-Boltzmann distributions, respectively, i.e. via Monte Carlo integration of the equation

$$
\begin{aligned}
k(T)= & \frac{\nu_{T}}{\left(k_{\mathrm{B}} T\right)^{2} Q} \sum_{v j}(2 j+1) \mathrm{e}^{-\varepsilon_{v j} / k_{\mathrm{B}} T} \\
& \times \int_{0}^{\infty} \mathrm{d} E_{c} E_{c} \mathrm{e}^{-E_{c} / k_{\mathrm{B}} T} \int_{0}^{\infty} \mathrm{d} b b P_{v j}^{\text {react }}\left(E_{c}, b\right)
\end{aligned}
$$

where $\nu_{T}=\left(8 k_{\mathrm{B}} T / \pi \mu\right)^{1 / 2}$ is the average thermal relative speed of the reactants at temperature $T$ ( $\mu$ being their reduced mass), $E_{c}$ is the collision energy and $b$ the impact parameter. $(v, j)$ are the vibrational and rotational quantum numbers of the molecular partner, $\varepsilon_{v j}$ its rovibrational energy and $Q$ its partition function. $P_{v j}^{\text {react }}\left(E_{c}, b\right)$ is the initial state-selected, total reaction probability at energy $E_{c}$ and impact parameter $b$.

\section{Ground state reactions}

Collisions between ground-state species $\mathrm{LiH}^{+}+\mathrm{H}$ are expected to be well described by the classical capture model. ${ }^{37}$ The main issue here is therefore the branching ratio governing the dissociation of the collision "complex", even though the potential well of this system is too shallow to allow formation of any long-lived collision complexes. Apart from inelastic scattering, dissociation may produce $\mathrm{H}_{2}+\mathrm{Li}^{+}$if it proceeds along the molecular hydrogen channel, and $\mathrm{LiH}^{+}+\mathrm{H}$ along the $\mathrm{H}$-exchange channel. These channels are exoergic or neutral and thus always energetically open. At energies of a few tenths of $\mathrm{eV}$, depending on the $\mathrm{LiH}^{+}$initial rovibrational state, dissociation along the break-up channel becomes possible, thereby complicating the picture. In order to check the reliability of the capture model, and to define appropriate 
maximum values for the impact parameter, we first computed opacity functions (i.e. the probability $P(b)$ of a given outcome as a function of the impact parameter $b$ ) for a number of initial rovibrational states of the $\mathrm{LiH}^{+}$molecule and of collision energies. The results are reported in Fig. 2, for $(v, j)=(0,0)$, $(1,0)$ and $(0,15)$ at collision energies of 50, 100 and $500 \mathrm{meV}$, along with the predictions of the capture model. In this model "capture" of the projectile occurs whenever the impact parameter is less than a critical value $b_{c}=\left(2 \alpha / E_{c}\right)^{1 / 4}$, where $\alpha$ represents the spherical polarizability of the neutral partner. In our case, $\alpha$ is the hydrogen atom polarizability, i.e. $9 / 2$ a.u. It is clear from Fig. 2 that the computed opacity functions at low collision energies are constant up to a given, energy-dependent maximum value which closely matches that predicted by the capture model. At higher collision energies, on the other hand, the behavior of $P(b)$ is more complicated and shows some structure arising from the finite size of the molecular target, i.e. a long molecule $\left(r_{e} \simeq 2 \AA\right)$ with its center of mass positioned very close to the $\mathrm{Li}$ end. For example, it seems that molecular formation occurs with some preference for insertion of the projectile hydrogen atom into the $\mathrm{Li}-\mathrm{H}$ bond (see top row in Fig. 2). The important point, however, is the fact that collision induced dissociation only roughly follows the prediction of a capture model, and it may occur at rather large impact parameters, especially if the target is rotationally excited. The reason is that for this weakly bound molecule rotational excitation is effective in increasing the molecular size. Based on the data reported in Fig. 2 it is not surprising that the
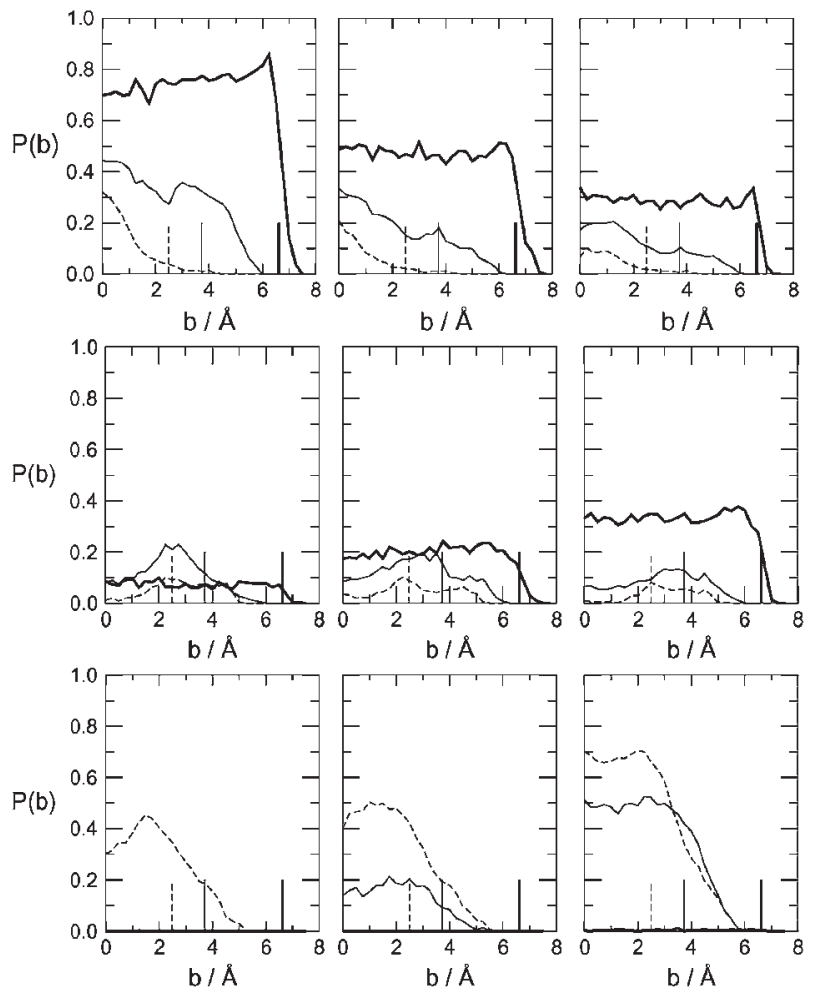

Fig. 2 Opacity functions for selected initial rovibrational states $(v, j)$ of $\mathrm{LiH}^{+},(v, j)=(0,0),(1,0)$ and $(0,15)$, on the left, middle and right panels, respectively. From top to bottom for (i) $\mathrm{H}_{2}$ formation, (ii) $\mathrm{H}$ exchange and (iii) collision induced dissociation. Bold, thin and dashed lines for $E_{\mathrm{col}}=10,100,500 \mathrm{meV}$. Vertical lines mark $b_{c}\left(E_{c}\right)$ values.
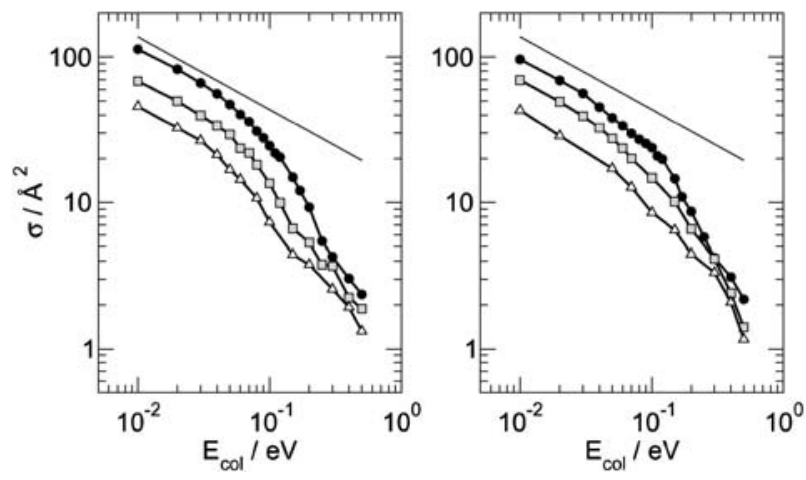

Fig. 3 Initial state-selected, integral cross sections of $\mathrm{H}_{2}$ formation for different initial rovibrational states $(v, j)$ of $\mathrm{LiH}^{+}$. Left panel for $j=0$ and $v=0$ (circles), 1 (squares) and 2 (triangles). Right panel: for $v=0$ and $j=5$ (circles), 10 (squares) and 15 (triangles). Solid lines indicate the capture model cross section for this reaction. Symbol sizes approximately match the estimated error bars.

computed cross-sections are reasonably described by the capture-model expression $\sigma_{c}\left(E_{c}\right)=\pi\left(2 \alpha / E_{c}\right)^{1 / 2}$, especially at low energy and for ground-state target molecules. This is shown in Fig. 3 for the cross sections of the $\mathrm{H}_{2}$ formation reaction. We considered separately vibrational and rotational excitation of the target molecules: on the left panel of Fig. 3 we show the cross-sections for $j=0$ and $v=0,1$ and 2, whereas on the right panel those for $v=0$ and $j=5,10$ and 15. It is evident from Fig. 3 that (i) the capture cross-section represents a true upper bound to the computed cross sections, in accordance with the results of Fig. 2, and (ii) vibrational and rotational excitation of the target molecule both have an inhibiting effect on the reaction. This is mainly due to the mirroring effect that molecular excitation has on the competing processes, as we show in Fig. 4 and 5, where we report the cross sections of the hydrogen exchange reaction and of the collision induced dissociation of $\mathrm{LiH}^{+}$, respectively. It is clear from Fig. 4 and 5 that, below the threshold to the break-up channel, molecular excitation (both vibrational and rotational) results in an increased cross-section for the exchange channel, whereas at higher energies we observe a weaker dependence on the initial state since the main effect of molecular excitation is to increase the probability of molecular dissociation (see Fig. 5). In this respect it is worth noting that rotational excitation can be even
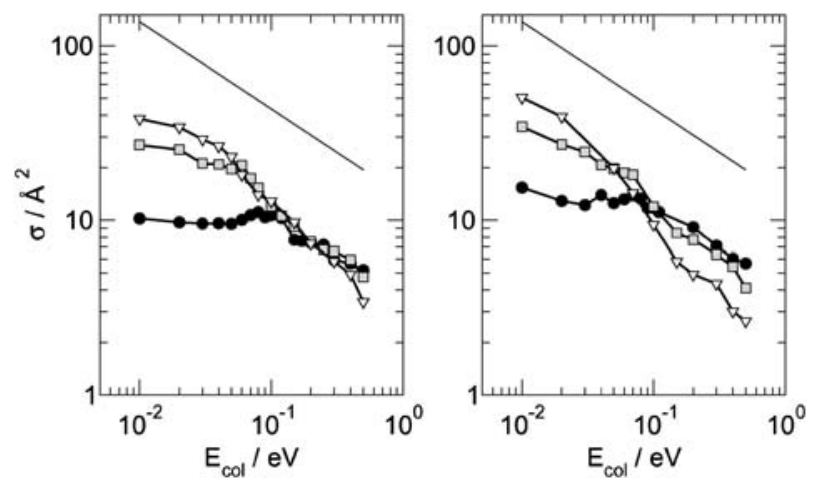

Fig. 4 Hydrogen exchange state-selected cross sections for different initial rovibrational states $(v, j)$ of $\mathrm{LiH}^{+}$. Left panel for $j=0$ and $v=0,1,2$. Right panel: for $v=0$ and $j=5,10,15$. Lines and symbols as in Fig. 3. 

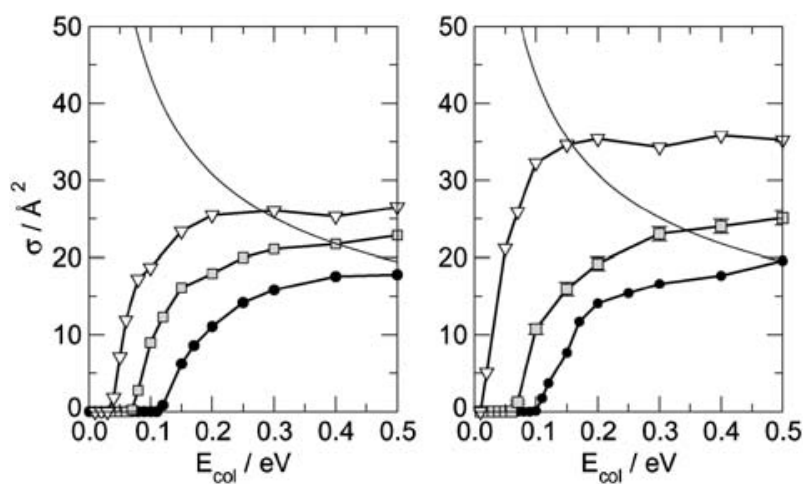

Fig. 5 CID state-selected cross sections for different initial rovibrational states $(v, j)$ of $\mathrm{LiH}^{+}$. Left panel for $j=0$ and $v=0,1,2$. Right panel: for $v=0$ and $j=5,10,15$. Lines and symbols as in Fig. 3.

more effective than vibrational one in promoting dissociation. As already observed before, this is due to the weak bond of the $\mathrm{LiH}^{+}$molecule which is strongly influenced by centrifugal distortions. A closer look at the dissociation mechanism reveals that about one third of the dissociation products is actually produced as a metastable intermediate (see Fig. 6), i.e. with one of the three possible pairs of atoms trapped by the centrifugal barrier with an energy content higher than the dissociation energy. This means that part of the dissociation - and in particular when the energy is just above the threshold-occurs through a two step mechanism, metastable molecule formation and decay. This behavior was already found in classical and quantum studies of a closely related process, namely the collision induced desorption of atoms stuck on surfaces. ${ }^{38,39}$ Of course, in classical mechanics these quasi-bound molecules are indefinitely stable but we can distinguish them from the truly bound molecules by analyzing their energy contents, and therefore we considered them when computing the total collision induced dissociation cross sections. Finally we consider the thermal rate constants for the above processes. We computed them by direct sampling of the Boltzmann distribution for the internal + translational energy since at
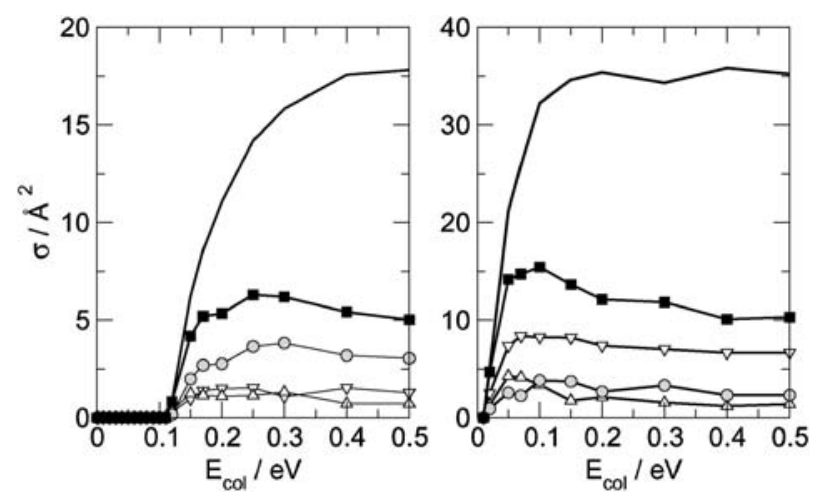

Fig. $6 \mathrm{LiH}^{+}+\mathrm{H}$ collision induced dissociation cross sections with their contribution due to quasi-bound molecules formation. Left and right panels for $(v, j)=(0,0)$ and $(0,15)$, respectively. Metastable species are indicated with triangles up $\left(\mathrm{LiH}_{a}{ }^{+}\right)$, triangles down $\left(\mathrm{LiH}_{b}{ }^{+}\right)$ and circles $\left(\mathrm{H}_{2}\right)$. Squares for total metastable contributions. Symbol sizes approximately match the estimated error bars.

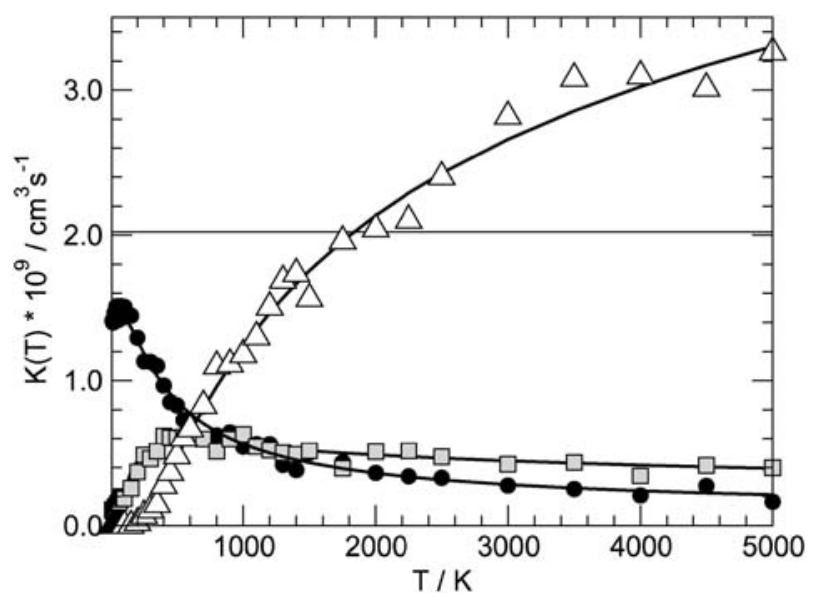

Fig. 7 Thermal rate constants for $\mathrm{H}_{2}$ formation (circles), hydrogen exchange (squares) and collision induced dissociation (triangles). Solid lines are simple fit to the data, that are: $k(T) * 10^{9} /\left(\mathrm{cm}^{3} \mathrm{~s}^{-1}\right)=$ $A_{0}-A_{1}(T / \mathrm{K})^{a} \mathrm{e}^{-b /(T / \mathrm{K})}$, with $A_{0}=1.46, A_{1}=1.24, a=0.01$ and $b=390.66$, for $\mathrm{H}_{2}$ formation, and $k(T) * 10^{9} /\left(\mathrm{cm}^{3} \mathrm{~s}^{-1}\right)=$ $A_{0}(T / \mathrm{K})^{a} \mathrm{e}^{-b /(T / \mathrm{K})}$, with $A_{0}=4.65(0.65), a=-0.29(0.21)$ and $b=165.05$ (808.34) for $\mathrm{H}$ exchange (CID). The horizontal line marks the value of the capture rate constant for this system. Symbol sizes approximately match the estimated error bars.

all but very low temperatures a large number of rovibrational states of the $\mathrm{LiH}^{+}$molecule is appreciably populated. The results of these calculation in the temperature range $10-5000 \mathrm{~K}$ are shown in Fig. 7, along with the capture rate constant, $k_{c}=$ $2 \pi(\alpha / \mu)^{1 / 2}=2.02 \times 10^{-9} \mathrm{~cm}^{3} \mathrm{~s}^{-1}\left(\mu\right.$ being the $\mathrm{LiH}^{+}-\mathrm{H}$ reduced mass). In accordance with the above discussion we can see from Fig. 7 that the capture estimate is reasonably good except in a medium-to-high temperature regime where the collision induced dissociation rate is itself larger than the capture value. It is clear from this figure that for $T \geq 800 \mathrm{~K}$ the collision induced dissociation of $\mathrm{LiH}^{+}$by hydrogen atoms is quite efficient; at lower temperature $\mathrm{LiH}^{+}$depletion is possible $\left(k \approx 10^{-9} \mathrm{~cm}^{3} \mathrm{~s}^{-1}\right)$ through hydrogen formation and therefore, overall, at typical interstellar conditions $\mathrm{LiH}^{+}$ molecules are expected to be rapidly destroyed by collisions with the abundant hydrogen atoms. In concluding this section it is worth noting that the rate constants shown in Fig. 7 have to be multiplied by the probability that the two reaction partners are found in the singlet state, i.e. 1/4. In the triplet state the two partners are expected to experience a repulsive interaction, because of the unfavorable spin coupling. This means that collision induced dissociation may still occur in this electronic state, and thus its total rate is expected to be similar to the one shown in Fig. 7. On the other hand, molecular hydrogen formation and hydrogen exchange cannot occur in the triplet state and their total rates are $1 / 4$ of the ones shown in Fig. 7.

\section{Excited state reactions}

In this section we consider the reactions occurring on the first electronically excited state of the $\mathrm{LiH}_{2}{ }^{+}$system, namely the exoergic $\mathrm{LiH}+\mathrm{H}^{+} \rightarrow \mathrm{Li}+\mathrm{H}_{2}{ }^{+}$reaction and its reverse. These reactions have already been considered in previous 
quantum ${ }^{23-25}$ and QCT $^{25}$ studies. The authors of ref. 24 found surprisingly that the exoergic hydrogen molecular ion forming reaction was dynamically activated, as is evident from the thresholds appearing in both cross sections and rate constants reported in ref. 24. More recently, Bulut et al. ${ }^{25}$ performed quantum and quasi-classical calculations for $J=0$ and found that, apart from the resonance structure, quantum and classical calculations agree very well with each other at the state-to-state level and showed no evidence of any threshold as long as vibrationally ground-state products were considered. The authors of ref. 25 further computed integral cross sections and from them thermal rate constants which were found in reasonably good agreement with a capture model. Since the detailed analysis of ref. 25 is already available (and an analogous study is being published for the reverse reaction ${ }^{40}$ ) we limit ourselves to show that our QCT results fully agree with those of ref. 25. This is shown in Fig. 8 where we report the $J=0$ reaction probabilities of ref. 25 along with our QCT results. For clarity, only the quantum results of Bulut et al. are shown, since it was already shown that they agreed with the classical ones, ${ }^{25}$ as is confirmed by our calculations. It is clear from this figure that (i) classical calculations are adequate in describing this reaction, as long as the resonance structure is of no concern, and (ii) the reaction does not show any threshold. This means that no threshold is expected in the exoergic $\mathrm{LiH}+\mathrm{H}^{+} \rightarrow \mathrm{Li}+\mathrm{H}_{2}^{+}$rate constant. The resonance structure occurs because of formation of long-lived collision complexes, which complicate the dynamics. Since they arise from the presence of deep wells in the reaction coordinate, they have their classical analogue, as is shown, for example, in Fig. 9 where we report a typical "trapped" trajectory for such a collision, in terms of the $\mathrm{HH}$ and the shortest $\mathrm{LiH}$ distance. It is clear from that figure that such complexes survive several picoseconds before breaking-up into one of the possible channels $\left(\mathrm{H}_{2}{ }^{+}\right.$formation, hydrogen exchange and non-reactive channel), thereby requiring long propagation times when integrating the equations of motion (both in classical and in quantum mechanics). On the other hand, this is the ideal
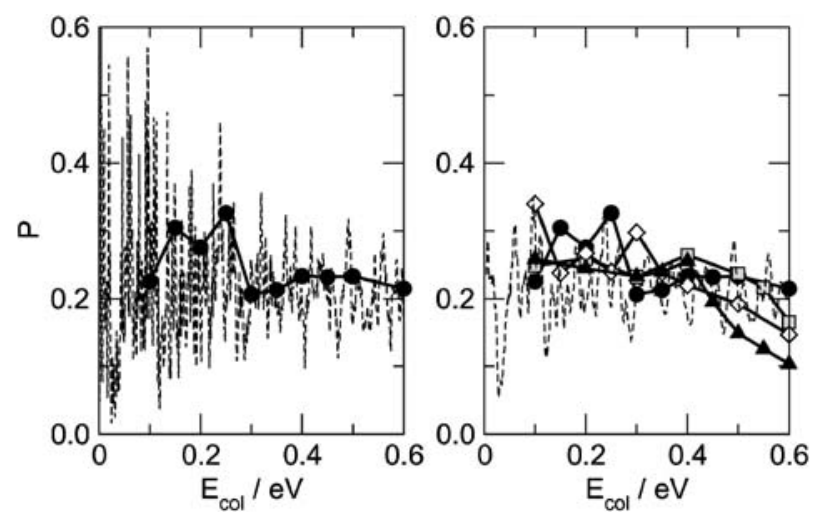

Fig. 8 Total $J=0$ probabilities for the reaction $\mathrm{LiH}(0, j)+\mathrm{H}^{+} \rightarrow \mathrm{Li}$ $+\mathrm{H}_{2}{ }^{+}$. On the left panel $j=0$ QCT results from this work (circles) are compared with quantum wave packet calculations of ref. 25 . On the right panel these quantum results for $j=0$ are averaged over a small energy interval for clarity (dashed line), and compared with QCT results for $j=0,1,2,3$ (circles, squares, diamonds, triangles).

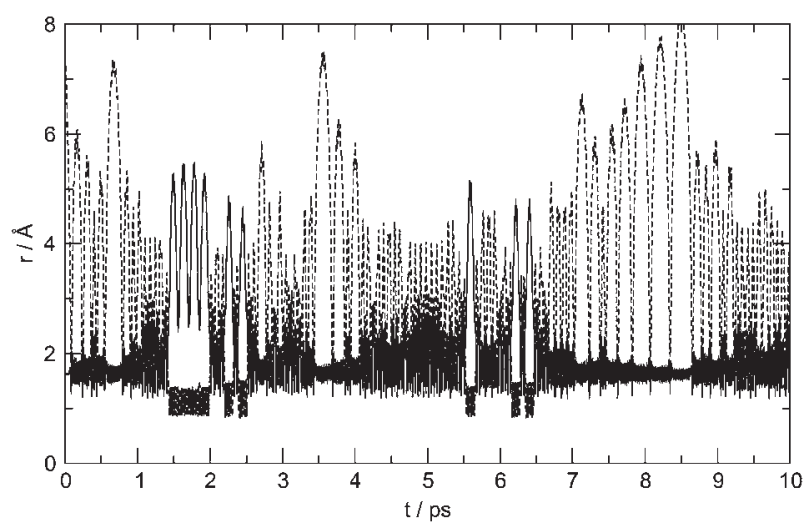

Fig. 9 A typical long-lived reactive trajectory in $\mathrm{LiH}+\mathrm{H}^{+}$collisions. Solid and dashed lines for the $\mathrm{HH}$ and the shortest $\mathrm{LiH}$ internuclear distance.

situation for application of statistical theories. ${ }^{41}$ As in the ground-state case, we computed thermal rate constants for this and its reverse reaction by direct sampling of the internal + translational energy distribution. Because of the long-range nature of the charge-dipole interaction, and in addition to the above mentioned need of long propagation times, very large impact parameters had to be considered, which made the calculation of the direct reaction rather expensive (and somewhat inaccurate). Therefore, for all but the lowest temperatures, we considered the shorter-ranged reverse reaction and applied the detailed balance to get information on the exoergic reaction, using the equilibrium constant and half the rate of total $\mathrm{LiH}$ formation. The results of these calculations are reported in Fig. 10, along with the equilibrium rate constant for the reaction $\mathrm{LiH}+\mathrm{H}^{+} \rightarrow \mathrm{Li}+\mathrm{H}_{2}{ }^{+}$. The capture rate constant for this reaction $k_{c}=2 \pi(\alpha / \mu)^{1 / 2}=5.15 \times$ $10^{-9} \mathrm{~cm}^{3} \mathrm{~s}^{-1}$ (where now $\alpha=29.0$ a.u. is the spherical polarizability of the $\mathrm{LiH}$ molecule $\dagger$ and $\mu$, as before, is the $\mathrm{LiH}-\mathrm{H}$ reduced mass) is in reasonably good agreement with the computed data, and the previous estimate by Stancil et $a l^{42}\left(k=1 \times 10^{-9} \mathrm{~cm}^{3} \mathrm{~s}^{-1}\right)$ is incidentally even better. It is worth noting that such agreement is not a priori obvious (especially at low temperature where steering of the projectile atom can occur) since the dominant long-range contribution to the potential is due to the strongly anisotropic chargedipole interaction. This means that such behavior must be due to rotational excitation of the target molecule (either thermal or during the collision), which may then show a rotationally averaged, spherical, charge-induced dipole potential as the dominant term at long range. In conclusion, this section comments on the electronic spin state. Specifically, in the case of $\mathrm{Li}+\mathrm{H}_{2}{ }^{+}$collisions, the probability that the reactants follow the singlet state adiabat (the one considered in this work) is $1 / 4$. In the overall triplet state the valence electron of the lithium atom couples unfavorably with the $\mathrm{H}_{2}{ }^{+}$electron, and no reaction is expected to occur. Then, the total $\mathrm{LiH}$ formation rate is $1 / 4$ of the one shown in Fig. 10.

$\dagger$ This is the spherical polarizability at the LiH equilibrium geometry as computed by the finite field technique at the CASSCF-MRCI level of theory of ref. 15 . 


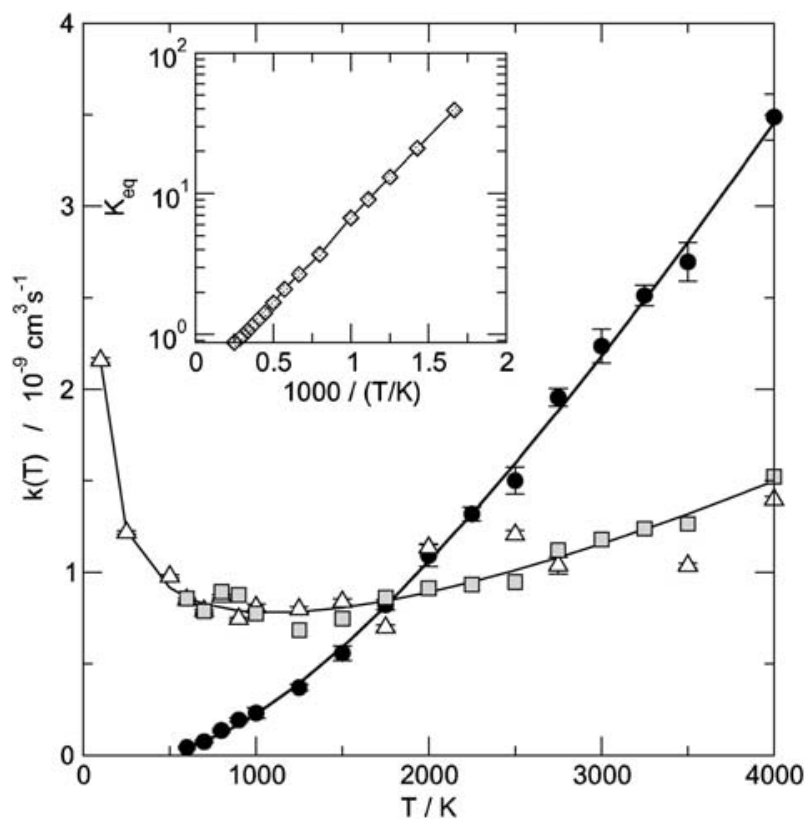

Fig. 10 Rate constant of $\mathrm{Li}+\mathrm{H}_{2}{ }^{+} \rightarrow \mathrm{LiH}+\mathrm{H}^{+}$(black circles) and its reverse reaction (gray squares) as obtained by detailed balance. Bold lines represent a simple fit to the data, $k(T) * 10^{9} /\left(\mathrm{cm}^{3} \mathrm{~s}^{-1}\right)=A_{0}(T / \mathrm{K})^{a} \mathrm{e}^{-b /(T / \mathrm{K})}$, with $A_{0}=2.88 \times 10^{-4}, a=1.18$ and $b=1469.83$ for the first reaction, and $k(T) * 10^{9} /\left(\mathrm{cm}^{3} \mathrm{~s}^{-1}\right)=A_{0}(T / \mathrm{K})^{a}+A_{1}(T / \mathrm{K})^{b} \mathrm{e}^{-c /(T / \mathrm{K})}$, with $A_{0}=$ 17.77, $a=-0.50, A_{1}=6.54 \times 10^{-6}, b=1.45$ and $c=-432.06$ for the second one. Triangles are results for direct calculations of the reverse reaction. The inset shows the equilibrium constant of the reaction $\mathrm{LiH}+$ $\mathrm{H}^{+} \rightarrow \mathrm{Li}+\mathrm{H}_{2}{ }^{+}$as a function of temperature. Symbol sizes approximately match the estimated error bars.

\section{Summary}

In this work we have applied the QCT method to compute the rate constants for a number of reactions involving the two lowest lying electronic states of the $\mathrm{LiH}_{2}{ }^{+}$system, using accurate $a b$ initio potential energy surfaces. The analytical expressions for these rate constants (see Fig. 7 and 10) are collected in Table 2 . They have been properly corrected for the corresponding spin factors, when needed, according to the discussions in sections 3 and 4 . We found that the calculated rate constants have values in reasonably good agreement with those estimated by the capture model, i.e. they are all of the order $\simeq 10^{-9} \mathrm{~cm}^{3} \mathrm{~s}^{-1}$, usually employed in the evolutionary models of the early universe chemistry. In detail, we provided improved analytical expressions for the rates of these reactions and, in addition, for those processes which lacked any estimate. In particular, the thermal rate constant for the

Table 2 Suggested values for the rate constants of the ionic reactions in the lithium chemistry considered in this work

\begin{tabular}{ll}
\hline Reaction & $k / 10^{-9} \mathrm{~cm}^{3} \mathrm{~s}^{-1}$ \\
\hline $\mathrm{LiH}^{+}+\mathrm{H} \rightarrow \mathrm{Li}^{+}+\mathrm{H}_{2}$ & $0.36-0.31(T / \mathrm{K})^{0.01} \mathrm{e}^{-390.7 /(T / \mathrm{K})}$ \\
$\mathrm{LiH}^{+}+\mathrm{H} \rightarrow \mathrm{Li}^{+}+\mathrm{H}+\mathrm{H}$ & $0.65(T / \mathrm{K})^{0.21} \mathrm{e}^{-808.3 /(T / K)}$ \\
$\mathrm{LiH}+\mathrm{H}^{+} \rightarrow \mathrm{Li}+\mathrm{H}_{2}^{+}$ & $17.77(T / \mathrm{K})^{-0.501}+6.54 \times 10^{-6}$ \\
& $(T / \mathrm{K})^{1.45} \mathrm{e}^{432.1 /(T / \mathrm{K})}$ \\
$\mathrm{Li}+\mathrm{H}_{2}^{+} \rightarrow \mathrm{LiH}+\mathrm{H}^{+}$ & $7.2 \times 10^{-5}(T / \mathrm{K})^{1.18} \mathrm{e}^{-1469.8 /(T / \mathrm{K})}$ \\
\hline
\end{tabular}

collision induced dissociation suggests that $\mathrm{LiH}^{+}$molecules are rapidly depleted by collisions with the abundant $\mathrm{H}$ atoms, thereby reducing the overall $\mathrm{LiH}$ abundance.

\section{Acknowledgements}

We thank the C.I.L.E.A. supercomputing center for having allowed the use of its computational facilities and Luis Bañares for having provided the results of ref. 25 .

\section{References}

1 T. Prodanović and B. D. Fields, Astrophys. J., 2004, 616, L115.

2 N. Yoshida, T. Abel, L. Hernquist and N. Sugiyama, Astrophys. J., 2003, 592, 645.

3 D. Puy, G. Alecian, J. Le Bourlot, J. Leorat and G. P. des Forets, Astron. Astrophys., 1993, 267, 337.

4 S. Lepp and P. C. Stancil, Oxford Science Publications, 1999, ch. 3, p. 37; International series on astronomy and astrophysics.

5 M. Tegmark, J. Silk, M. J. Rees, A. Blanchard, T. Abel and F. Palla, Astrophys. J., 1997, 474, 1.

6 T. Abel, G. Bryan and M. Norman, Astrophys. J., 2000, 540, 39.

7 S. Lepp, P. C. Stancil and A. Dalgarno, Mem. Soc. Astron. Ital., 1998, 69(2), 331.

8 D. Galli and F. Palla, Astron. Astrophys., 1998, 335, 403.

9 S. Lepp, P. C. Stancil and A. Dalgarno, J. Phys. B, 2002, 35(10), R57.

10 E. Bodo, F. A. Gianturco and R. Martinazzo, Phys. Rep., 2003, 384, 85.

11 R. Maoli, F. Melchiorri and D. Tosti, Astrophys. J., 1994, 458, 372.

12 E. Bodo, F. A. Gianturco, R. Martinazzo, A. Forni, A. Famulari and M. Raimondi, J. Phys. Chem., 2000, 104, 11972.

13 E. Bodo, F. A. Gianturco, R. Martinazzo and M. Raimondi, J. Phys. Chem. A, 2001, 105, 10986.

14 R. Martinazzo, E. Bodo, F. A. Gianturco and M. Raimondi, Chem. Phys., 2003, 287, 335.

15 R. Martinazzo, G. F. Tantardini, E. Bodo and F. A. Gianturco, J. Chem. Phys., 2003, 119, 11241.

16 E. Bodo, F. A. Gianturco, R. Martinazzo and M. Raimondi, Chem. Phys., 2001, 271, 309.

17 R. Martinazzo, A. Famulari, M. Raimondi, E. Bodo and F. A. Gianturco, J. Chem. Phys., 2001, 2917.

18 C. Sanz, E. Bodo and F. A. Gianturco, Chem. Phys., 2005, 314, 135.

19 W. P. Kraemer and V. Špirko, Chem. Phys., 2006, 330, 190.

20 A. J. Page and E. I. von Nagy-Felsobuki, J. Phys. Chem. A, 2007, 111, 4478.

21 C. D. Thompson, C. Emmeluth, B. L. J. Poad, G. H. Weddle and E. J. Bieske, J. Chem. Phys., 2006, 125, 044310.

22 C. Emmeluth, B. L. J. Poad, C. D. Thompson and E. J. Bieske, J. Chem. Phys., 2007, 126, 204309.

23 F. Gögtas, J. Chem. Phys., 2005, 123, 244301.

24 F. Gögtas, Int. J. Quantum Chem., 2006, 106, 1979.

25 N. Bulut, J. F. Castillo, F. J. Aoiz and L. Bañares, Phys. Chem. Chem. Phys., 2008, 10, 821.

26 F. Gögtas, Chem. Phys. Lett., 2005, 407, 298.

27 S. Akpinar and F. Gögtas, THEOCHEM, 2006, 765, 115

28 M. Karplus, R. N. Porter and R. D. Sharma, J. Chem. Phys., 1965, 43(9), 3259.

29 G. F. Tantardini and M. Simonetta, Surf. Sci., 1981, 105, 517.

30 G. F. Tantardini and M. Simonetta, Chem. Phys. Lett., 1982, 87(5), 420.

31 V. I. Pazzi and G. F. Tantardini, Surf. Sci., 1997, 377-379, 572.

32 V. I. Pazzi, P. H. T. Philipsen, E. J. Baerends and G. F. Tantardini, Surf. Sci., 1999, 443, 1.

33 R. Martinazzo, S. Assoni, G. Marinoni and G. F. Tantardini, J. Chem. Phys., 2004, 120, 8761.

34 I. Pino, Quasiclassical dynamics of astrophysically and catalytically relevant systems, $\mathrm{PhD}$ thesis, University of Milan, 2006.

35 C. W. Gear, Numerical Initial Value Problems in Ordinary Differential Equations, Prentice-Hall, Upper Saddle River, NJ, USA, 1971.

36 D. T. Colbert and W. H. Miller, J. Chem. Phys., 1992, 96, 1982. 
37 P. M. Langevin, Ann. Chim. Phys., 1905, 5, 245.

38 R. Martinazzo and G. F. Tantardini, J. Phys. Chem. A, 2005, 42, 9379.

39 R. Martinazzo and G. F. Tantardini, J. Chem. Phys., 2006, 124, 124702.

40 L. Bañares, personal communication.
41 S. C. Smith, in Modern Trends in Chemical Reaction DynamicsExperiments and Theory (Part I), ed. X. Yang and K. Liu, Advanced Series in Physical Chemistry, World Scientific, 2004, vol. 14, p. 291.

42 P. C. Stancil, S. Lepp and A. Dalgarno, Astrophys. J., 1996, 458, 401. 